\title{
Positive solutions of functional-differential systems via the vector version of Krasnoselskii's fixed point theorem in cones
}

\section{SORIN BUDIŞAN and RADU PRECUP}

\section{ABSTRACT.}

We study the existence of positive solutions of the functional-differential system

$$
\left\{\begin{array}{l}
u_{1}^{\prime \prime}(t)+a_{1}(t) f_{1}\left(u_{1}(g(t)), u_{2}(g(t))\right)=0, \\
u_{2}^{\prime \prime}(t)+a_{2}(t) f_{2}\left(u_{1}(g(t)), u_{2}(g(t))\right)=0
\end{array}\right.
$$

$(0<t<1)$, subject to linear boundary conditions. We prove the existence of at least one positive solution by using the vector version of Krasnoselskii's fixed point theorem in cones.

"BABEŞ-BOLYAI" UNIVERSITY

DEPARTMENT OF MATHEMATICS

400084 Cluj-NAPOCA, ROMANIA

E-mail address: sorinbudisan@yahoo.com

E-mail address: $r$.precup@math.ubbcluj.ro

Received: 09.11.2010; In revised form: 20.03.2011; Accepted: 30.06.2011

2000 Mathematics Subject Classification. 34B18, 34K10.

Key words and phrases. Positive solution, boundary value problem, fixed point, cone. 